\title{
THE WEIGHTED EULER-MACLAURIN FORMULA FOR A SIMPLE INTEGRAL POLYTOPE*
}

\author{
JOSÉ AGAPITO ${ }^{\dagger}$ AND JONATHAN WEITSMAN ${ }^{\ddagger}$
}

\begin{abstract}
We give an Euler-Maclaurin formula with remainder for the weighted sum of the values of a smooth function on the integral points in a simple integral polytope. Our work generalizes the formula obtained in [KSW2].
\end{abstract}

Key words. Simple polytope, Euler-Maclaurin formula, Hirzebruch genus

AMS subject classifications. Primary 65B15, 52B20

1. Introduction. The Euler-Maclaurin formula computes the sum of the values of a function $f$ over the integer points in an interval in terms of the integral of $f$ over variations of that interval. Khovanskii and Pukhlikov [KP1, KP2] and Kantor and Khovanskii $[\mathrm{KK}]$ generalized the classical Euler-Maclaurin formula to higher dimensional convex polytopes $\Delta$ which are integral and regular. This formula was generalized to simple integral polytopes by Cappell and Shaneson [CS1, CS2], and subsequently by Guillemin $[\mathrm{G}]$ and by Brion-Vergne [BV]. These generalizations involve corrections to Khovanskii's formula when the simple polytope is not regular. These formulas are exact formulas, valid for sums of exponential or polynomial functions. With the use of the $\mathbf{L}$ class associated to the signature operator, as in [CS1, CS2], Karshon, Sternberg and Weitsman gave an Euler-Maclaurin formula with remainder for a weighted sum of the values of an arbitrary smooth function on the lattice points in a simple integral polytope [KSW1, KSW2].

The purpose of this paper is to give a generalization of the Euler-Maclaurin formula with remainder of [KSW2], to allow for more general weightings, including the ordinary, unweighted sum. To do this, we use the Hirzebruch formal power series $\chi_{q}(S)[\mathrm{H}]$ and the weighted polar decomposition of $[\mathrm{A}]$.

\section{Weighted Euler-Maclaurin in one dimension.}

Weighted sums in one dimension. Let $q$ be any complex number and $f(x)$ be any function on the real line. For any integers $a<b$, define

$$
\sum_{[a, b]}^{q} f:=q f(a)+f(a+1)+\ldots+f(b-1)+q f(b) .
$$

Similarly, for a ray $[a, \infty)$ with $f$ having compact support

$$
\sum_{[a, \infty)}^{q} f:=q f(a)+f(a+1)+\ldots
$$

SO

$$
\sum_{[a, b]}^{q} f=\sum_{[a, \infty)}^{q} f-\sum_{[b, \infty)}^{1-q} f
$$

\footnotetext{
*Received January 7, 2004; accepted for publication November 5, 2004. This work was partially supported by National Science Foundation Grant DMS 99/71914.

${ }^{\dagger}$ Department of Mathematics, University of California, Santa Cruz, CA 95064, USA (jarpepe@ math.ucsc.edu).

‡Department of Mathematics, University of California, Santa Cruz, CA 95064, USA (weitsman@ math.ucsc.edu).
} 
If $q=1$ this is the ordinary sum; if $q=1 / 2$ this is the weighted sum of [KSW2].

Euler-Maclaurin formulas. The classical Todd function is defined by

$$
\operatorname{Td}(S)=\frac{S}{1-\mathrm{e}^{-S}}=1-b_{1} S+\sum_{n=1}^{\infty} \frac{b_{2 n}}{(2 n) !} S^{2 n},
$$

where $b_{n}$ is the $n$-th Bernoulli number (we are using the conventions in [B]). Similarly, the $\mathbf{L}$ function is given by

$$
\mathbf{L}(S)=\frac{S / 2}{\tanh (S / 2)}=1+\sum_{n=1}^{\infty} \frac{b_{2 n}}{(2 n) !} S^{2 n} .
$$

Both $\mathbf{T d}(S)$ and $\mathbf{L}(S)$ are convergent power series for $|S|<2 \pi$.

The Hirzebruch function is defined by

$$
\chi_{q}(S)=q \mathbf{T d}(S)+(1-q) \mathbf{T d}(-S)=1+\left(q-\frac{1}{2}\right) S+\sum_{n=1}^{\infty} \frac{b_{2 n}}{(2 n) !} S^{2 n} .
$$

It is related to $\mathbf{T d}$ and to $\mathbf{L}$ by

$$
\chi_{q}(S)=(q-1) S+\mathbf{T d}(S)=\left(q-\frac{1}{2}\right) S+\mathbf{L}(S) .
$$

Thus if $q=1$ we have $\chi_{1}(S)=\mathbf{T d}(S)$ and if $q=1 / 2$, we get $\chi_{1 / 2}(S)=\mathbf{L}(S)$.

Let $f$ be a compactly supported function on the real line of class $C^{m}$ where $m>1$. The standard Euler-Maclaurin formula with remainder for a ray can be written (see for instance $[\mathrm{B}])$

$$
\sum_{[a, \infty)} f=\left.\mathbf{T d}^{2 k}\left(\frac{\partial}{\partial h}\right) \int_{a-h}^{\infty} f(x) d x\right|_{h=0}+R_{m}^{a}(f),
$$

where $k=\lfloor m / 2\rfloor$, where $\mathbf{T d}^{2 k}(S)$ denotes the truncation of the power series $\mathbf{T d}(S)$ at the $2 k$-th term, and where the remainder $R_{m}^{a}(f)$ is given by

$$
R_{m}^{a}(f)=(-1)^{m-1} \int_{a}^{\infty} P_{m}(x) f^{(m)}(x) d x \quad \text { with } \quad P_{m}(x)=\frac{B_{m}(\{x\})}{m !} .
$$

Here, $B_{m}(x)$ is the $m$ th Bernoulli polynomial (see $[\mathrm{B}]$ ) and $\{x\}=x-\lfloor x\rfloor$ is the fractional part of $x$. Moreover, the function $P_{m}(x)$ is given by

$$
P_{2 k}(x)=(-1)^{k-1} \sum_{n=1}^{\infty} \frac{2 \cos 2 \pi n x}{(2 \pi n)^{2 k}}
$$

if $m=2 k$, and by

$$
P_{2 k+1}(x)=(-1)^{k-1} \sum_{n=1}^{\infty} \frac{2 \sin 2 \pi n x}{(2 \pi n)^{2 k+1}}
$$

if $m=2 k+1$. 
Similarly, denoting the truncation of the power series $\chi_{q}$ at the $2 k$-th term by $\chi_{q}^{2 k}$, we have a weighted Euler-Maclaurin formula with remainder for a ray,

$$
\sum_{[a, \infty)}^{q} f=\left.\chi_{q}^{2 k}\left(\frac{\partial}{\partial h}\right) \int_{a-h}^{\infty} f(x) d x\right|_{h=0}+R_{m}^{a}(f),
$$

where $f$ is a compactly supported function.

Combining (9) and (3) we get

$$
\sum_{[a, b]}^{q} f=\left.\chi_{q}^{2 k}\left(\frac{\partial}{\partial h_{1}}\right) \int_{a-h_{1}}^{\infty} f(x) d x\right|_{h_{1}=0}-\left.\chi_{1-q}^{2 k}\left(\frac{\partial}{\partial h_{2}}\right) \int_{b-h_{2}}^{\infty} f(x) d x\right|_{h_{2}=0}+R_{m}^{[a, b]}(f)
$$

where

$$
R_{m}^{[a, b]}(f)=R_{m}^{b}(f)-R_{m}^{a}(f)=(-1)^{m-1} \int_{a}^{b} P_{m}(x) f^{(m)}(x) d x .
$$

Given the fact that $\chi_{q}^{2 k}(S)$ is a polynomial whose constant term is 1, we can write this as

$$
\sum_{[a, b]}^{q} f=\left.\chi_{q}^{2 k}\left(\frac{\partial}{\partial h_{1}}\right) \chi_{1-q}^{2 k}\left(\frac{\partial}{\partial h_{2}}\right)\left(\int_{a-h_{1}}^{\infty} f(x) d x-\int_{b-h_{2}}^{\infty} f(x) d x\right)\right|_{h_{1}=h_{2}=0}+R_{m}^{[a, b]}(f)
$$

To make further progress, we note the following symmetry property.

$$
\chi_{q}^{2 k}(S)=\chi_{1-q}^{2 k}(-S) .
$$

(To see this, observe that $\chi_{q}^{2 k}(S)$ is a polynomial with constant coefficients whose constant term is 1 , whose linear term is $(q-1 / 2) S$ (see (5)), and whose other terms are all of even degree independent of $q$.)

Thus we obtain the following result:

Theorem 1 (Euler-Maclaurin with remainder for intervals). Let $f(x)$ be a function with $m>1$ continuous derivatives and let $k=\lfloor m / 2\rfloor$. Then

$$
\sum_{[a, b]}^{q} f=\left.\chi_{q}^{2 k}\left(\frac{\partial}{\partial h_{1}}\right) \chi_{q}^{2 k}\left(\frac{\partial}{\partial h_{2}}\right) \int_{a-h_{1}}^{b+h_{2}} f(x) d x\right|_{h_{1}=h_{2}=0}+R_{m}^{[a, b]}(f) .
$$

Note that our argument applies to functions $f$ of compact support. However, for a general function $f$ of type $C^{m}$, the theorem remains true: We need only multiply $f$ by a smooth function of compact support which is identically one in a neighborhood of $[a, b]$. of $f$ :

If $f$ is a polynomial, Theorem 1 becomes exact when $m$ is greater than the degree

Corollary 14. Let $f$ be a polynomial. Then

$$
\sum_{[a, b]}^{q} f=\left.\chi_{q}\left(\frac{\partial}{\partial h_{1}}\right) \chi_{q}\left(\frac{\partial}{\partial h_{2}}\right) \int_{a-h_{1}}^{b+h_{2}} f(x) d x\right|_{h_{1}=h_{2}=0} .
$$


Twisted weighted Euler-Maclaurin for a ray. Consider the "twisted weighted sum"

$$
\sum_{n \geq 0}^{q} \lambda^{n} f(n)=q f(0)+\sum_{n=1}^{\infty} \lambda^{n} f(n)
$$

where $\lambda \neq 1$ is a root of unity, say, of order $N$.

Let $Q_{m, \lambda}(x)$ be distributions successively defined by

$$
Q_{0, \lambda}(x)=-\sum_{n \in \mathbb{Z}} \lambda^{n} \delta(x-n)
$$

and

$$
\frac{d}{d x} Q_{m, \lambda}(x)=Q_{m-1, \lambda}(x) \text { and } \quad \int_{0}^{N} Q_{m, \lambda}(x) d x=0 .
$$

These distributions appear in [KSW2].

Now, define the polynomial

$$
\mathbf{N}_{q}^{k, \lambda}(S)=\left(q+\frac{\lambda}{1-\lambda}\right) S+Q_{2, \lambda}(0) S^{2}+Q_{3, \lambda}(0) S^{3}+\cdots+Q_{k, \lambda}(0) S^{k},
$$

for a root of unity $\lambda \neq 1$. When $q=1 / 2$, we get the polynomial $\mathbf{M}^{k, \lambda}(S)$ defined in [KSW2]. Since $\mathbf{N}_{q}^{k, \lambda}(S)$ and $\mathbf{M}^{k, \lambda}(S)$ differ by $(q-1 / 2) S$, adding this term gives the following generalization of Proposition 23 in [KSW2].

Proposition 17. Let $k>1$ and let $f \in C_{c}^{k}(\mathbb{R})$. Then

$$
\sum_{n \geq 0}^{q} \lambda^{n} f(n)=\left.\mathbf{N}_{q}^{k, \lambda}\left(\frac{\partial}{\partial h}\right) \int_{-h}^{\infty} f(x) d x\right|_{h=0}+(-1)^{k-1} \int_{0}^{\infty} Q_{k, \lambda}(x) f^{(k)}(x) d x
$$

As in [KSW2], we have the following symmetry property

$$
\mathbf{N}_{1-q}^{m, 1 / \lambda}(S)=\mathbf{N}_{q}^{m, \lambda}(-S)
$$

REMARK 20. For $\lambda=1$, we define

$$
\mathbf{N}_{q}^{k, 1}(S)=\chi_{q}^{2\lfloor k / 2\rfloor}(S) \quad \text { and } \quad Q_{k, 1}=P_{k},
$$

then (18) boils down to (9). So, (18) also holds for $\lambda=1$. Notice that if $\lambda \neq 1$ then $\mathbf{N}_{q}^{k, \lambda}(S)$ is a multiple of $S$, and that if $\lambda=1$ then $\mathbf{N}_{q}^{k, \lambda}(S)=1+$ a multiple of $S$. Property (19) continues to hold for $\lambda=1$ because of the symmetry property (12). Finally, if $\lambda=1$, we get the truncation $\mathbf{T d}^{k}(S)$ of $\mathbf{T} \mathbf{d}(S)$ for $q=1$, and the corresponding truncation at $k$ of $\mathbf{T d}(-S)$ for $q=0$. These two expressions differ by $S$. 
3. Simple polytopes and finite groups associated to them. In this section we recall various combinatorial and group-theoretic facts about simple polytopes which will be needed in our proof of the weighted Euler-Maclaurin formula with remainder. Most of this material is taken from [A] and [KSW2] and is included for completeness.

Let $H_{i}=\left\{x \mid\left\langle u_{i}, x\right\rangle+\mu_{i} \geq 0, \mu_{i} \in \mathbb{R}\right\}$ be a half space in $\mathbb{R}^{n}$, where $u_{i} \in \mathbb{R}^{n *}$ for $i=1, \ldots, d$. A compact convex polytope $\Delta$ in $\mathbb{R}^{n}$ is a compact set which can be written as the intersection of finitely many half-spaces

$$
\Delta=H_{1} \cap \ldots \cap H_{d},
$$

with the smallest possible $d$, so that the $H_{i}$ 's are uniquely determined up to permutation. We order them arbitrarily. The facets (codimension one faces) of $\Delta$ are

$$
\sigma_{i}=\Delta \cap \partial H_{i} \quad, \quad i=1, \ldots, d .
$$

The vertices of $\Delta$ are all possible codimension $\mathrm{n}$ faces obtained by intersections of facets.

A polytope is called integral if its vertices are in the lattice $\mathbb{Z}^{n}$; it is called simple if exactly $n$ edges emanate from each vertex; it is called regular if, additionally, the edges emanating from each vertex lie along lines which are generated by a $\mathbb{Z}$-basis of the lattice $\mathbb{Z}^{n}$.

For each vertex $v$ of $\Delta$, let $I_{v} \subset\{1, \ldots, d\}$ encode the set of facets that contain $v$, so that

$$
i \in I_{v} \quad \text { if and only if } \quad v \in \sigma_{i} .
$$

The vector $u_{i} \in \mathbb{R}^{n *}$ can be thought of as the inward normal to the $i$ th facet of $\Delta$; a-priori it is determined up to multiplication by a positive number. If the polytope $\Delta$ is integral, then the $u_{i}$ 's can be chosen to belong to the dual lattice $\mathbb{Z}^{n *}$, and we can fix our choice of the $u_{i}$ 's by imposing the normalization condition that the $u_{i}$ 's be primitive lattice elements, that is, that no $u_{i}$ can be expressed as a multiple of a lattice element by an integer greater than one.

Assume that $\Delta$ is simple, so that each vertex is the intersection of exactly $n$ facets. For each $i \in I_{v}$, there exists a unique edge at $v$ which does not belong to the facet $\sigma_{i}$; choose any vector $\alpha_{i, v}$ in the direction of this edge. These vectors form a dual basis to the inward normal vectors $u_{i}$ 's and are uniquely determined when the polytope is integral and the $u_{i}$ 's are normalized in the sense explained above.

A "polarizing vector" $\xi \in \mathbb{R}^{n *}$ is a vector such that $\left\langle\xi, \alpha_{i, v}\right\rangle$ is non-zero for all vertices $v$ and all edges $i$ emanating from $v$. A choice of a polarizing vector $\xi$ determine polarized edge vectors $\alpha_{i, v}^{\sharp}$ defined by

$$
\alpha_{i, v}^{\sharp}=\left\{\begin{array}{lll}
\alpha_{i, v} & \text { if }\left\langle\xi, \alpha_{i, v}\right\rangle<0, & \text { (unflipped) } \\
-\alpha_{i, v} & \text { if }\left\langle\xi, \alpha_{i, v}\right\rangle>0, & \text { (flipped) }
\end{array} .\right.
$$

Let $q$ be any complex number. For each $i \in I_{v}$ define

$$
q_{i, v}^{\sharp}=\left\{\begin{array}{cl}
q & \text { if } \alpha_{i, v}^{\sharp}=\alpha_{i, v} \\
1-q & \text { if } \alpha_{i, v}^{\sharp}=-\alpha_{i, v}
\end{array} .\right.
$$


The tangent cone to $\Delta$ at $v$ is

$$
\mathbf{C}_{v}=\{v+r(x-v) \mid r \geq 0, x \in \Delta\}=v+\sum_{i \in I_{v}} \mathbb{R}_{\geq 0} \alpha_{i, v} .
$$

Similarly the polarized tangent cone at $v$ is defined by

$$
\mathbf{C}_{v}^{\sharp}=v+\sum_{i \in I_{v}} \mathbb{R}_{\geq 0} \alpha_{i, v}^{\sharp} .
$$

A simple integral orthant $\mathbf{C}$ in $\mathbb{R}^{n}$ is the intersection of $n$ half-planes in general position,

$$
\mathbf{C}=H_{1} \cap \ldots \cap H_{n} \quad \text { with } \quad H_{i}=\left\{x \mid\left\langle u_{i}, x\right\rangle+\mu_{i} \geq 0, \mu_{i} \in \mathbb{R}\right\} \quad \text { for } \quad i=1, \ldots, n,
$$

where the $u_{i}$ 's are inward normals to the facets $\sigma_{i}=\mathbf{C} \cap \partial H_{i}$ of $\mathbf{C}$, which can be chosen to be primitive elements of the dual lattice $\mathbb{Z}^{n *}$, and whose vertex $v=\cap_{i=1}^{n}\{x \mid$ $\left.\left\langle u_{i}, x\right\rangle+\mu_{i}=0, \mu_{i} \in \mathbb{R}\right\}$ is in $\mathbb{Z}^{n}$. This implies that $\mu_{i} \in \mathbb{Z}$ for all $1 \leq i \leq n$. If $\alpha_{1}, \ldots, \alpha_{n}$ is the dual basis to the $u_{i}$ 's, that is,

$$
\left\langle u_{j}, \alpha_{i}\right\rangle= \begin{cases}1 & j=i \\ 0 & j \neq i\end{cases}
$$

then

$$
\mathbf{C}=v+\sum_{j=1}^{n} \mathbb{R}_{\geq 0} \alpha_{j} .
$$

We associate a complex number $q_{i}$ to each facet $\sigma_{i}$ of $\mathbf{C}$, and define the weighting function

$$
w(x)=\prod_{i \in I_{F}} q_{i},
$$

where $I_{F}$ denotes the set of facets in $\mathbf{C}$ meeting at the face $F$ (the smallest dimensional face in $\mathbf{C}$ containing $x$.) If $x$ is in the interior of $\mathbf{C}$, we set $w(x)=1$, and finally we set $w(x)=0$ if $x \notin \mathbf{C}$.

For a simple integral orthant, we consider the weighted sum

$$
\sum_{\mathbf{C} \cap \mathbb{Z}^{n}}^{\mathbf{q}} f=\sum_{x \in \mathbf{C} \cap \mathbb{Z}^{n}} w(x) f(x),
$$

where $\mathbf{q}$ denotes the $n$-tuple $\left(q_{1}, \ldots q_{n}\right)$ used in the definition of $w(x)$.

For a polytope, we consider the weighted sum

$$
\sum_{\Delta \cap \mathbb{Z}^{n}}^{q} f=\sum_{x \in \Delta \cap \mathbb{Z}^{n}} q^{c(x)} f(x),
$$

where $q$ is any complex number, and where $c(x)$ is the codimension of the smallest dimensional face in $\Delta$ containing $x$.

Given $q \in \mathbb{C}$, a cone $\mathbf{C}_{v}$ and a polarizing vector $\xi \in \mathbb{R}^{n *}$, we get the $n$-tuple $\mathbf{q}_{v}^{\sharp}:=\left(q_{1, v}^{\sharp}, \ldots, q_{n, v}^{\sharp}\right)$ where $q_{i, v}^{\sharp}$ is defined in (23). With this notation, the weighted polar decomposition of $[\mathrm{A}]$ is as follows. 
Theorem 2 ([A]). For any polarizing vector $\xi$, we have

$$
\sum_{\Delta \cap \mathbb{Z}^{n}}^{q} f=\sum_{v}(-1)^{\# v} \sum_{\mathbf{C}_{v}^{\sharp} \cap \mathbb{Z}^{n}}^{\mathbf{q}_{v}^{\sharp}} f,
$$

where we sum over the vertices $v$ of $\Delta$ and where $\# v$ is the number of edge vectors at $v$ that are "flipped" by the polarization process (22).

To obtain a weighted Euler-Maclaurin formula with remainder for simple integral polytopes, we associate certain finite groups to such polytopes. We recall some definitions and results from Section $\S 5$ in [KSW2].

Let us consider a simple integral orthant $\mathbf{C}$ with vertex $v \in \mathbb{Z}^{n}$, as given in (26). To $\mathbf{C}$ we associate the finite group

$$
\Gamma:=\mathbb{Z}^{n *} / \sum \mathbb{Z} u_{i}
$$

This group is trivial exactly if $\mathbf{C}$ is regular.

Now let $\Delta$ be a simple integral polytope in $\mathbb{R}^{n}$. For any face $F$ of $\Delta$, let $I_{F}$ denote the set of facets of $\Delta$ which meet at $F$. Because $\Delta$ is simple, the vectors $u_{i}$, for $i \in I_{F}$, are linearly independent. Let $N_{F} \subseteq \mathbb{R}^{n *}$ be the subspace

$$
N_{F}=\operatorname{span}\left\{u_{i} \mid i \in I_{F}\right\}
$$

To each face $F$ of $\Delta$ we associate a finite abelian group $\Gamma_{F}$. Explicitly, the lattice

$$
V_{F}=\sum_{i \in I_{F}} \mathbb{Z} u_{i} \subset N_{F}
$$

is a sublattice of $N_{F} \cap \mathbb{Z}^{n *}$ of finite index, and the finite abelian group associated to the face $F$ is the quotient

$$
\Gamma_{F}:=\left(N_{F} \cap \mathbb{Z}^{n *}\right) / V_{F} .
$$

If $F=v$ is a vertex, this is the same as the finite abelian group associated to the tangent cone $\mathbf{C}_{v}$ as in (31).

Let $E$ and $F$ be two faces of $\Delta$ with $F \subseteq E$. This inclusion implies that $I_{E} \subseteq I_{F}$, and hence $\Gamma_{E} \subseteq \Gamma_{F}$.

We define a subset $\Gamma_{F}^{b}$ of $\Gamma_{F}$ by

$$
\Gamma_{F}^{b}:=\Gamma_{F} \backslash \bigcup_{\text {faces } E \text { such that } E \supsetneq F} \Gamma_{E} .
$$

Then

$$
\Gamma_{v}=\bigsqcup_{\{F: v \in F\}} \Gamma_{F}^{b} .
$$

The map

$$
\lambda_{\gamma, j, v}:=e^{2 \pi i\left\langle\gamma, \alpha_{j, v}\right\rangle}, \quad \text { for } \gamma \in \Gamma_{v} \quad \text { and } j \in I_{v},
$$

is a well defined character and it is a root of unity. 
Claim 36 ([KSW2], Claim 61). If $\gamma \in \Gamma_{F}$ and $j \in I_{F}$, then $\lambda_{\gamma, j, v}$ is the same for all $v \in F$.

This allows us to define $\lambda_{\gamma, j, F}$ for $\gamma \in \Gamma_{F}$ and $j \in I_{F}$ such that

$$
\lambda_{\gamma, j, F}=\lambda_{\gamma, j, v} \quad \text { for } \gamma \in \Gamma_{F} \text { and } j \in I_{F} \text {, if } v \in F \text {. }
$$

Claim 37 ([KSW2], Claim 62). If $\gamma \in \Gamma_{F}$ and $j \in I_{v} \backslash I_{F}$ then $\lambda_{\gamma, j, v}$ is equal to one.

This allows us to define $\lambda_{\gamma, j, F}=1$ when $\gamma \in \Gamma_{F}$ and $j \in\{1, \ldots, d\} \backslash I_{F}$. Then

$$
\lambda_{\gamma, j, F}=\lambda_{\gamma, j, v} \quad \text { for } \gamma \in \Gamma_{F} \text { and } 1 \leq j \leq d, \text { if } v \in F
$$

and

$$
\lambda_{\gamma, j, F}=1 \quad \text { for } \gamma \in \Gamma_{F} \text { if } j \notin I_{F} .
$$

Claim 40 ([KSW2], Claim 65). If $\gamma \in \Gamma_{F}^{b}$ and $j \in I_{F}$, then $\lambda_{\gamma, j, F} \neq 1$.

4. Weighted Euler-Maclaurin with remainder for simple integral polytopes. Let $\Gamma$ be the finite group (31) associated to a simple integral orthant $\mathbf{C}$ (as defined in (26)) with vertex at $v \in \mathbb{Z}^{n}$. The map $\gamma \mapsto \mathrm{e}^{\langle\gamma, x\rangle}$ defines a character on $\Gamma$ ([KSW2], Lemma 52) whenever $x \in \sum \mathbb{Z} \alpha_{j}$, and this character is trivial if and only if $x \in \mathbb{Z}^{n}$.

By a theorem of Frobenius,

$$
\frac{1}{|\Gamma|} \sum_{\gamma \in \Gamma} \mathrm{e}^{2 \pi i\langle\gamma, x\rangle}= \begin{cases}1 & \text { if } x \in \mathbb{Z}^{n} \\ 0 & \text { if } x \notin \mathbb{Z}^{n}\end{cases}
$$

for all $x \in \sum \mathbb{Z} \alpha_{j}$. Then, for any vector $\mathbf{q}=\left(q_{1}, \ldots, q_{n}\right) \in \mathbb{C}^{n}$ and any function $f(x)$ compactly supported on $\mathbb{R}^{n}$,

$$
\begin{aligned}
\sum_{\mathbf{C} \cap \mathbb{Z}^{n}}^{\mathbf{q}} f & =\sum_{x}^{\mathbf{q}}\left(\frac{1}{|\Gamma|} \sum_{\gamma \in \Gamma} \mathrm{e}^{2 \pi i\langle\gamma, x\rangle}\right) f(x) \\
& =\frac{1}{|\Gamma|} \sum_{\gamma \in \Gamma} \sum_{x}^{\mathbf{q}} \mathrm{e}^{2 \pi i\langle\gamma, x\rangle} f(x)
\end{aligned}
$$

where we sum over all

$$
x=v+m_{1} \alpha_{1}+\ldots+m_{n} \alpha_{n},
$$

with the $m_{i}$ 's being non-negative integers.

The simple integral orthant $\mathbf{C}$ is the image of the standard orthant $\mathbf{O}=\prod_{i=1}^{n} \mathbb{R}_{\geq 0}$ in $\mathbb{R}^{n}$ under the affine map

$$
\left(t_{1}, \ldots, t_{n}\right) \mapsto v+\sum t_{i} \alpha_{i} .
$$

This map sends the lattice $\mathbb{Z}^{n}$ onto the lattice $\sum \mathbb{Z} \alpha_{j}$. Let us concentrate on one element $\gamma \in \Gamma$. Because $v \in \mathbb{Z}^{n}$, from (42) we get

$$
\mathrm{e}^{2 \pi i\langle\gamma, x\rangle}=\prod_{j=1}^{n} \lambda_{j}^{m_{j}} \quad \text { where } \quad \lambda_{j}=\mathrm{e}^{2 \pi i\left\langle\gamma, \alpha_{j}\right\rangle},
$$


so that the inner sum in (41) becomes

$$
\sum_{x}^{\mathbf{q}} \mathrm{e}^{2 \pi i\langle\gamma, x\rangle} f(x)=\sum_{m_{1} \geq 0}^{q_{1}} \lambda_{1}^{m_{1}} \cdots \sum_{m_{n} \geq 0}^{q_{n}} \lambda_{n}^{m_{n}} g\left(m_{1}, \ldots, m_{n}\right),
$$

where

$$
g\left(m_{1}, \ldots, m_{n}\right)=f\left(v+m_{1} \alpha_{1}+\ldots+m_{n} \alpha_{n}\right) .
$$

Given $q \in \mathbb{C}$ and $k>1$, we had the twisted remainder formula (see (18))

$$
\sum_{m \geq 0}^{q} \lambda^{m} g(m)=\left.\mathbf{N}_{q}^{k, \lambda}\left(\frac{\partial}{\partial h}\right) \int_{-h}^{\infty} g(t) d t\right|_{h=0}+(-1)^{k-1} \int_{0}^{\infty} Q_{k, \lambda}(t) g^{(k)}(t) d t
$$

for all compactly supported functions $g(x)$ of type $C^{k}$, where $k>1$, where $\lambda$ is a root of unity, and where $N_{q}^{k, \lambda}$ is a polynomial of degree $\leq k$.

Iterating this formula, the sum in (43) can be written as

$$
\begin{aligned}
\mathbf{N}_{q_{1}}^{k, \lambda_{1}}\left(\frac{\partial}{\partial h_{1}}\right) \int_{-h_{1}}^{\infty} \cdots \mathbf{N}_{q_{n}}^{k, \lambda_{n}}\left(\frac{\partial}{\partial h_{n}}\right) \int_{-h_{n}}^{\infty} g\left(t_{1}, \ldots, t_{n}\right) d t_{1} \cdots d t_{n} \\
+R_{\mathbf{q}, k}^{s t}\left(\lambda_{1}, \ldots, \lambda_{n} ; g\right) \\
=\prod_{i=1}^{n} \mathbf{N}_{q_{i}}^{k, \lambda_{i}}\left(\frac{\partial}{\partial h_{i}}\right) \int_{\mathbf{O}(h)} g\left(t_{1}, \ldots, t_{n}\right) d t_{1} \cdots d t_{n}+R_{\mathbf{q}, k}^{s t}\left(\lambda_{1}, \ldots, \lambda_{n} ; g\right),
\end{aligned}
$$

where

$$
\mathbf{O}\left(h_{1}, \ldots, h_{n}\right)=\left\{\left(t_{1}, \ldots, t_{n}\right) \mid t_{i} \geq-h_{i} \text { for all } i\right\}
$$

denotes the "dilated" standard orthant, and where the remainder is given by

$$
\begin{aligned}
R_{\mathbf{q}, k}^{s t}\left(\lambda_{1}, \ldots, \lambda_{n} ; g\right) & :=\sum_{I \subsetneq\{1, \ldots, n\}}(-1)^{(k-1)(n-|I|)} \\
& \left.\prod_{i \in I} \mathbf{N}_{q_{i}}^{k, \lambda_{i}}\left(\frac{\partial}{\partial h_{i}}\right) \int_{\mathbf{O}(h)} \prod_{i \notin I} Q_{k, \lambda_{i}}\left(t_{j}\right) \prod_{i \notin I} \frac{\partial^{k}}{\partial t_{i}^{k}} g\left(t_{1}, \ldots, t_{n}\right) d t_{1} \cdots d t_{n}\right|_{h=0}
\end{aligned}
$$

with

$$
g\left(t_{1}, \ldots, t_{n}\right)=f\left(v+t_{1} \alpha_{1}+\ldots+t_{n} \alpha_{n}\right) .
$$

Performing the change of variable given by the transformation

$$
L:\left(t_{1}, \ldots, t_{n}\right) \mapsto x=v+t_{1} \alpha_{1}+\ldots+t_{n} \alpha_{n},
$$

whose Jacobian is $1 /|\Gamma|$, and substituting back into (41), we get

$$
\sum_{\mathbf{C} \cap \mathbb{Z}^{n}}^{\mathbf{q}} f=\left.\sum_{\gamma \in \Gamma} \prod_{i=1}^{n} \mathbf{N}_{q_{i}}^{k, \lambda_{\gamma, i}}\left(\frac{\partial}{\partial h_{i}}\right) \int_{\mathbf{C}(h)} f(x) d x\right|_{h=0}+R_{\mathbf{q}, k}^{\mathbf{C}}(f),
$$


where $\mathbf{C}\left(h_{1}, \ldots, h_{n}\right)$ denotes the image of the "dilated" standard orthant $\mathbf{O}\left(h_{1}, \ldots, h_{n}\right)$ under the affine transformation $L$, and where the remainder is given by

$$
R_{\mathbf{q}, k}^{\mathbf{C}}(f):=\frac{1}{|\Gamma|} \sum_{\gamma \in \Gamma} R_{\mathbf{q}, k}^{s t}\left(\lambda_{\gamma, 1}, \ldots, \lambda_{\gamma, n} ; L^{*} f\right)
$$

where

$$
\lambda_{\gamma, j}:=\mathrm{e}^{2 \pi i\left\langle\gamma, \alpha_{j}\right\rangle}
$$

Now let $\Delta$ be a simple integral polytope, given by (21). Choose a polarizing vector for $\Delta$ and let $\mathbf{C}_{v}^{\sharp}$ denote the polarized tangent cones. We can consider the "dilated polytope" $\Delta\left(h_{1}, \ldots, h_{d}\right)$, which is obtained by shifting the $i$ th facet outward by a "distance" $h_{i}$. More precisely,

$$
\Delta(h)=\bigcap_{i=1}^{d}\left\{x \mid\left\langle u_{i}, x\right\rangle+\mu_{i}+h_{i} \geq 0\right\} \quad \text { where } \quad h=\left(h_{1}, \ldots, h_{d}\right) .
$$

Then $\Delta(h)$ is simple if $h$ is sufficiently small. The polar decomposition of $\Delta(h)$ involves "dilated orthants". However, dilating the facets of $\Delta$ outward results in dilating some facets of $\mathbf{C}_{v}^{\sharp}$ inward and some outward. Explicitly, for $i \in I_{v}=\left\{i_{1}, \ldots, i_{n}\right\}$, the inward normal vector to the $i$ th facet of $\mathbf{C}_{v}^{\sharp}$ is

$$
u_{i, v}^{\sharp}= \begin{cases}u_{i} & \text { if } \alpha_{i, v}^{\sharp}=\alpha_{i, v} \\ -u_{i} & \text { if } \alpha_{i, v}^{\sharp}=-\alpha_{i, v} .\end{cases}
$$

Hence, the dilated orthants that occur on the right hand side of the polar decomposition of $\Delta(h)$ are $\mathbf{C}_{v}^{\sharp}\left(h_{i_{1}, v}^{\sharp}, \ldots, h_{i_{n}, v}^{\sharp}\right)$, where

$$
h_{i, v}^{\sharp}= \begin{cases}h_{i} & \text { if } \alpha_{i, v}^{\sharp}=\alpha_{i, v} \\ -h_{i} & \text { if } \alpha_{i, v}^{\sharp}=-\alpha_{i, v} .\end{cases}
$$

Because the inward normals to the facets of $\mathbf{C}_{v}^{\sharp}$ are given by (47), the dual basis to these vectors is $\alpha_{i, v}^{\sharp}, i \in I_{v}$, and the roots of unity that appear in the Euler-Maclaurin formula for $\mathbf{C}_{v}^{\sharp}$ are then

$$
\lambda_{\gamma, i, v}^{\sharp}=\mathrm{e}^{2 \pi i\left\langle\gamma, \alpha_{i, v}^{\sharp}\right\rangle}= \begin{cases}\lambda_{\gamma, i, v} & \text { if } \alpha_{i, v}^{\sharp}=\alpha_{i, v} \\ \lambda_{\gamma, i, v}^{-1} & \text { if } \alpha_{i, v}^{\sharp}=-\alpha_{i, v} .\end{cases}
$$

Let $k>1$ be an integer. For any compactly supported function $f$ on $\mathbb{R}^{n}$ of type $C^{n k}$, we then get from (30) and (45)

$$
\begin{aligned}
& \sum_{\Delta \cap \mathbb{Z}^{n}}^{q} f=\sum_{v}(-1)^{\# v} \sum_{\mathbf{C}_{v}^{\sharp} \cap \mathbb{Z}^{n}}^{\mathbf{q}_{v}^{\sharp}} f \\
& =\sum_{v}(-1)^{\# v} \sum_{\gamma \in \Gamma_{v}} \prod_{j \in I_{v}=\left\{i_{1}, \ldots, i_{n}\right\}} \mathbf{N}_{\left.q_{j, v}^{k, \lambda_{\gamma, j, v}^{\sharp}}\left(\frac{\partial}{\partial h_{j, v}^{\sharp}}\right) \int_{\mathbf{C}_{v}^{\sharp}\left(h_{i_{1}, v}^{\sharp}, \ldots, h_{i_{n}, v}^{\sharp}\right)} f(x) d x\right|_{h=0}}+R_{q, k}^{\Delta}(f),
\end{aligned}
$$


where the remainder is given by

$$
R_{q, k}^{\Delta}(f):=\sum_{v}(-1)^{\# v} R_{\mathbf{q}_{v}^{\sharp}, k}^{C_{\sharp}^{\sharp}}(f)
$$

and where the $h_{i, v}^{\sharp}$ 's are given in (48). Note that either $h_{i, v}^{\sharp}=h_{i}, \lambda_{\gamma, i, v}^{\sharp}=\lambda_{\gamma, i, v}$ and $q_{i, v}^{\sharp}=q$, or $h_{i, v}^{\sharp}=-h_{i}, \lambda_{\gamma, i, v}^{\sharp}=\lambda_{\gamma, i, v}^{-1}$ and $q_{i, v}^{\sharp}=1-q$. By the symmetry property (19), we have

$$
\mathbf{N}_{q_{i, v}^{\sharp}}^{k, \lambda_{\gamma, i, v}^{\sharp}}\left(\frac{\partial}{\partial h_{i, v}^{\sharp}}\right)=\mathbf{N}_{q}^{k, \lambda_{\gamma, i, v}}\left(\frac{\partial}{\partial h_{i}}\right) .
$$

Now, for $j \notin I_{v}$, because $\lambda_{\gamma, j, v}=1$ (see (39)), we have $\mathbf{N}_{q}^{k, \lambda_{\gamma, j, v}}\left(\frac{\partial}{\partial h_{j}}\right)=$ $1+$ powers of $\frac{\partial}{\partial h_{j}}$. Also for $j \notin I_{v}$, the cone $C_{v}^{\sharp}\left(h_{i_{1}, v}^{\sharp}, \ldots, h_{i_{n}, v}^{\sharp}\right)$ is independent of $h_{j}$. These facts together with Formula (52) imply that (50) is equal to

$$
\left.\sum_{v}(-1)^{\# v} \sum_{\gamma \in \Gamma_{v}} \prod_{j=1}^{d} \mathbf{N}_{q}^{k, \lambda_{\gamma, j, v}}\left(\frac{\partial}{\partial h_{j}}\right) \int_{C_{v}^{\sharp}\left(h_{i_{1}, v}^{\sharp}, \ldots, h_{i_{n}, v}^{\sharp}\right)} f(x) d x\right|_{h=0}+R_{q, k}^{\Delta}(f) .
$$

Because $\lambda_{\gamma, j, F}=\lambda_{\gamma, j, v}$ whenever $v \in F$ (see (38)), we can define

$$
\mathbf{N}_{q, \gamma, F}^{k}=\prod_{j=1}^{d} \mathbf{N}_{q}^{k, \lambda_{\gamma, j, F}}\left(\frac{\partial}{\partial h_{j}}\right) \quad \text { for } \gamma \in \Gamma_{F}
$$

and we have

$$
\mathbf{N}_{q, \gamma, F}^{k}=\mathbf{N}_{q, \gamma, v}^{k} \quad \text { whenever } \gamma \in \Gamma_{F} \text { and } v \in F,
$$

where we identify $\gamma \in \Gamma_{F}$ with its image under the inclusion map $\Gamma_{F} \hookrightarrow \Gamma_{v}$.

Then (53) is equal to

$$
\begin{array}{r}
\left.\quad \sum_{v}(-1)^{\# v} \sum_{\gamma \in \Gamma_{v}} \mathbf{N}_{q, \gamma, v}^{k} \int_{C_{v}^{\sharp}\left(h_{i_{1}}^{\sharp}, \ldots, h_{i_{n}}^{\sharp}\right)} f(x) d x\right|_{h=0}+R_{q, k}^{\Delta}(f) \\
=\left.\sum_{F} \sum_{\gamma \in \Gamma_{F}^{b}} \mathbf{N}_{q, \gamma, F}^{k} \sum_{v \in F}(-1)^{\# v} \int_{C_{v}^{\sharp}\left(h_{i_{1}}^{\sharp}, \ldots, h_{i_{n}}^{\sharp}\right)} f(x) d x\right|_{h=0}+R_{q, k}^{\Delta}(f),
\end{array}
$$

by (34) and (55). In the interior summation we may now add similar summands that correspond to $v \notin F$. These summands make a zero contribution to (56) for the following reason. If $v \notin F$ then there exists $i \in I_{F} \backslash I_{v}$. Because $i \notin I_{v}$, the cone $C_{v}^{\sharp}\left(h_{i_{1}, v}^{\sharp}, \ldots h_{i_{n}, v}^{\sharp}\right)$ is independent of $h_{i}$. So it is enough to show that $\mathbf{N}_{q, \gamma, F}^{k}$ is a multiple of $\frac{\partial}{\partial h_{i}}$. But because $\gamma \in \Gamma_{F}^{b}$ and $i \in I_{F}$, we have $\lambda_{\gamma, i, F} \neq 1$. (See Claim 40.) By Remark 20, this implies that $\mathbf{N}_{q}^{k, \lambda_{\gamma, i, F}}\left(\frac{\partial}{\partial h_{i}}\right)$, which is one of the factors in $\mathbf{N}_{q, \gamma, F}^{k}$, 
is a multiple of $\frac{\partial}{\partial h_{i}}$. Hence, $(56)$ is equal to

$$
\begin{gathered}
\left.\sum_{F} \sum_{\gamma \in \Gamma_{F}^{b}} \mathbf{N}_{q, \gamma, F}^{k} \sum_{\text {all } v}(-1)^{\# v} \int_{C_{v}^{\sharp}\left(h_{i_{1}, v}^{\sharp}, \ldots, h_{i_{n}, v}^{\sharp}\right)} f(x) d x\right|_{h=0}+R_{q, k}^{\Delta}(f) \\
=\left.\sum_{F} \sum_{\gamma \in \Gamma_{F}^{b}} \mathbf{N}_{q, \gamma, F}^{k} \int_{\Delta(h)} f(x) d x\right|_{h=0}+R_{q, k}^{\Delta}(f) .
\end{gathered}
$$

We have therefore proved our main result:

Theorem 3. Let $\Delta$ be a simple integral polytope in $\mathbb{R}^{n}$. Let $k>1$ and let $f \in C_{c}^{n k}\left(\mathbb{R}^{n}\right)$ be a compactly supported function on $\mathbb{R}^{n}$. Choose a polarizing vector for $\Delta$. Then

$$
\sum_{\Delta \cap \mathbb{Z}^{n}}^{q} f=\left.\sum_{F} \sum_{\gamma \in \Gamma_{F}^{b}} \mathbf{N}_{q, \gamma, F}^{k} \int_{\Delta(h)} f(x) d x\right|_{h=0}+R_{q, k}^{\Delta}(f)
$$

where $\mathbf{N}_{q, \gamma, F}^{k}$ are differential operators defined in (54) and where the remainder $R_{q, k}^{\Delta}(f)$ is given by equation (51). Moreover, the differential operators $\mathbf{N}_{q, \gamma, F}^{k}$ are of order $\leq k$ in each of the variables $h_{1}, \ldots, h_{d}$. Also, the remainder can be expressed as a sum of integrals over orthants of bounded periodic functions times various partial derivatives of $f$ of order no less than $k$ and no more than $k n$. This remainder is independent of the choice of polarization and is a distribution supported on the polytope $\Delta$.

In particular, if $q=1 / 2$, we get Theorem 2 of [KSW2], while if $q=1$, we have a formula for the ordinary, unweighted sum. Notice that if $q=0$, we also have an unweighted sum but only over the interior lattice points in the polytope.

5. Estimates on the remainder and an exact Euler-Maclaurin formula for polynomials. In order to derive a formula for polynomials from Theorem 3 , we first require an estimate on the remainder term $R_{q, k}^{\Delta}(f)$. Returning to the definition of functions $Q_{m, \lambda}(x)$ in [KSW2], we see that $Q_{m, \lambda}(x)$ is a periodic function on $\mathbb{R}$. It follows that $Q_{m, \lambda}(x)$ is bounded. Since the operators $\mathbf{N}_{q}^{k, \lambda}\left(\frac{\partial}{\partial h}\right)$ are differential operators of order $k$, the definition of the remainder $R_{\mathbf{q}, k}^{s t}\left(\lambda_{1}, \ldots, \lambda_{n} ; f\right)$ (see (44)) shows that

$$
\left|R_{\mathbf{q}, k}^{s t}\left(\lambda_{1}, \ldots, \lambda_{n} ; f\right)\right| \leq K_{\mathbf{q}} \cdot \sup _{\left\{j_{1}, \ldots, j_{n}\right\}}\left|\partial_{1}^{j_{1}} \ldots \partial_{n}^{j_{n}} f\right|_{L_{1}\left(\mathbb{R}^{n}\right)}
$$

where the supremum is taken over all $n$-tuples $\left\{j_{1}, \cdots, j_{n}\right\}$ with $k \leq j_{1}+\cdots+j_{n} \leq n k$.

The definition of $R_{q, k}^{\Delta}$ and equation (58) then give the same estimate for the remainder on the polytope, of course with a different constant.

Proposition 59. The remainder term in Theorem 3 can be estimated by

$$
\left|R_{q, k}^{\Delta}(f)\right| \leq K_{q}(k, \Delta) \cdot \sup _{\left\{j_{1}, \ldots, j_{n}\right\}}\left|\partial_{1}^{j_{1}} \ldots \partial_{n}^{j_{n}} f\right|_{L_{1}\left(\mathbb{R}^{n}\right)},
$$

where the supremum is taken over all $n$-tuples $\left\{j_{1}, \cdots, j_{n}\right\}$ with $k \leq j_{1}+\cdots+j_{n} \leq n k$.

The estimate in Proposition 59 implies 
Proposition 60. Let $p$ be a polynomial on $\mathbb{R}^{n}$, and choose $k \geq \operatorname{deg} p+n+1$. Then

$$
\sum_{\Delta \cap \mathbb{Z}^{n}}^{q} p=\left.\sum_{F} \sum_{\gamma \in \Gamma_{F}^{b}} \mathbf{N}_{q, \gamma, F}^{k} \int_{\Delta(h)} p(x) d x\right|_{h=0}
$$

Corollary 61. Let $p$ be a polynomial and suppose that the polytope $\Delta$ is regular. Then

$$
\sum_{\Delta \cap \mathbb{Z}^{n}}^{q} p=\left.\prod_{i=1}^{d} \chi_{q}\left(\frac{\partial}{\partial h}_{i}\right) \int_{\Delta(h)} p(x) d x\right|_{h=0}
$$

\section{REFERENCES}

[A] J. AgAPITo, A weighted version of quantization commutes with reduction principle for a toric manifold. Preprint: arXiv:math.SG/0307318.

[B] N. Bourbaki, Functions d'Une Variable Réele, Chapitre VI: Développments Tayloriens Géneralisés, Formule Summatoire d'Euler-Maclaurin (1951).

[BV] M. Brion And M. Vergne, Lattice points in simple polytopes, Jour. Amer. Math. Soc., 10 (1997), pp. 371-392.

[CS1] S. E. Cappell And J. L. Shaneson, Genera of algebraic varieties and counting lattice points, Bull. A. M. S., 30 (1994), pp. 62-69.

[CS2] S. E. CAPpell AND J. L. Shaneson, Euler-Maclaurin expansions for lattices above dimension one, C. R. Acad. Sci. Paris Sér. I Math., 321 (1995), pp. 885-890.

[G] V. Guillemin, Riemann-Roch for toric orbifolds, J. Diff. Geom., 45 (1997), pp. 53-73.

[H] F. Hirzebruch, Topological Methods in Algebraic Geometry, Springer, 1966.

[KK] J. M. Kantor And A. G. KhovanskiI, Une application du théorème de Riemann-Roch combinatoire au polynôme d'Ehrhart des polytopes entiers de $R^{d}$, C. R. Acad. SciParis Sér. I Math., 317:5 (1993), pp. 501-507.

[KSW1] Y. Karshon, S. Sternberg, and J. Weitsman, The Euler-Maclaurin formula for simple integral polytopes, Proc. Nat. Acad. Sci., 100:2 (2003), pp. 426-433.

[KSW2] Y. Karshon, S. Sternberg, And J. Weitsman, Euler-Maclaurin with remainder for a simple integral polytope, Preprint: arXiv:math.CO/0307125v1.

[KP1] A. G. Khovanskil And A. V. Pukhlikov, Finitely additive measures of virtual polytopes, Algebra and Analysis, 4 (1992), pp. 161-185; translation in St. Petersburg Math. J., 4:2 (1993), pp. 337-356.

[KP2] A. G. Khovanski And A. V. Pukhlikov, The Riemann-Roch theorem for integrals and sums of quasipolynomials on virtual polytopes, Algebra and Analysis, 4 (1992), pp. 188-216, translation in St. Petersburg Math. J., 4 (1993), pp. 789-812. 
